\title{
Planejamento de sucessão de lideranças em enfermagem: caminhos para sua elaboração*
}

\author{
Nursing leadership succession planning: paths for elaboration \\ Planificación de la sucesión de liderazgos en enfermería: caminos para su elaboración
}

Como citar este artigo:

Nogueira ALG, Munari DB, Sousa ET, Ribeiro LCM. Nursing leadership succession planning: paths for elaboration. Rev Esc Enferm USP. 2021;55:e03758. https://doi.org/10.1590/S1980-220X2020022103758

\section{Alyne Leite Gomes Nogueira ${ }^{1}$ \\ Denize Bouttelet Munari ${ }^{2}$ \\ Elyana Teixeira Sousa ${ }^{3}$ \\ Luana Cássia Miranda Ribeiro²}

* Extraído da tese: "Planejamento de sucessão: uma ferramenta para potencializar a liderança em enfermagem", Faculdade de Enfermagem, Universidade Federal de Goiás, 2018.

${ }^{1}$ Universidade Federal de Goiás, Faculdade de Enfermagem, Programa de Pós-Graduação em Enfermagem, Goiânia, GO, Brasil.

${ }^{2}$ Universidade Federal de Goiás, Faculdade de Enfermagem, Goiânia, GO, Brasil.

${ }^{3}$ Universidade Federal de Mato Grosso, Instituto de Saúde Coletiva, Cuiabá, MT, Brasil.

\section{ABSTRACT}

Objective: To describe the construction of a nursing leadership succession plan for a hospital based on the appreciative reflection of nurses. Method: Descriptive exploratory study with a qualitative approach carried out with nurses in a federal public teaching hospital. Data collection was carried out in two stages. The first was an individual interview, and the second was group mediation conducted based on the 4D Cycle and the assumptions of Appreciative Inquiry. Descriptive analysis of the findings was carried out. Results: The data showed a highly qualified team of different generations, concerned with succession planning based on scientific evidence, involving teams and carried out carefully for nurses at different stages of their professional careers. Conclusion: The principles of Appreciative Inquiry allowed the construction of a succession plan to enhance leadership development actions in the hospital. The collaborative environment between generations was valued and planning focused on participatory management was created in the process. This aspect can profoundly change the institution's internal policy in several areas and point out ways to implement succession planning in different contexts.

\section{DESCRIPTORS}

Nursing Administration Research; Leadership; Education, Nursing, Continuing; Health Workforce; Nurse Administrators; Qualitative Research.

\section{Autor correspondente:}

Elyana Teixeira Sousa

Av. das Palmeiras, s/n, Condomínio

Rio Claro, casa 73, Jardim Imperial

CEP 78075-901 - Cuiabá, MT, Brasil

elyanasousa@hotmail.com
Recebido: 30/05/2020

Aprovado: 01/01/2021 


\section{INTRODUÇÃO}

Enfermeiros que desempenham papel de líderes, em geral, não receberam educação formal sobre liderança ${ }^{(1)}$. Aqueles com conhecimentos clínicos e eficácia comprovada no processo de cuidar são promovidos e vão adquirindo habilidades para a liderança, sem apoio institucional. Isso, nem sempre, garante-lhes eficiência ao desempenhar esta função ${ }^{(2)}$.

A crescente necessidade de serviços para suprir as demandas globais relacionadas à saúde e o papel estratégico que os enfermeiros ocupam no sistema de saúde exigem que as organizações preparem uma nova geração de enfermeiros com capacidade para liderar equipes de alta performance ${ }^{(3)}$. Esse processo envolve a criação de programas formais de desenvolvimento de liderança e de preparação de enfermeiros para assumirem esse papel $^{(4)}$.

O Planejamento de Sucessão (PS) é uma das maneiras de elaborar projetos para o desenvolvimento institucional ${ }^{(5)}$ e se define como um processo de identificação e preparação de profissionais para a liderança, por meio de mentoring, de coaching e de rotação de cargos, para substituir membros-chave das organizações. É um processo baseado na antecipação de necessidades de liderança, para garantir que os cargos-chave sejam preenchidos internamente, por vacância de cargos por aposentadoria, conflito interno, licença de saúde e/ou outros ${ }^{(6)}$.

Dentre os benefícios citados na literatura em relação à implantação do PS, destacam-se: a possibilidade de aumentar a autopercepção de liderança e a competência gerencial dos enfermeiros ${ }^{(6-8)}$; o incentivo do desenvolvimento pessoal e profissional para o favorecimento da continuidade da liderança; e o suporte para progressão na carreira ${ }^{(5,7,9)}$.

A literatura especializada sobre o tema aponta que a aplicação do PS requer apoio organizacional sistemático, com plano estratégico para desenvolver a força de trabalho da organização, de articulação e de avaliação de competências de liderança desejadas, com a identificação das lacunas futuras baseadas nas promoções e na projeção de aposentadorias ${ }^{(7)}$. Por isso, o PS de lideranças deve ser pensado para cada realidade ${ }^{(4-6)}$.

$\mathrm{Na}$ área de enfermagem, o PS facilita a identificação de novas demandas para a renovação da força de trabalho profissional ${ }^{(10-11)}$, sendo útil para garantir a formação de líderes e o avanço da profissão e da assistência à saúde ${ }^{(11)}$. Por consequência, garante a continuidade do patrimônio intelectual construído por várias gerações de enfermeiros.

Embora a liderança seja uma competência essencial para os enfermeiros, evidências apontam que a formação acadêmica não tem sido suficiente para o seu alcance ${ }^{(2,12)}$; as organizações de saúde dão pouca atenção ao desenvolvimento de líderes ${ }^{(13)}$, e a preparação de enfermeiros para a sucessão de lideranças constitui-se em um problema a ser enfrentado ${ }^{(5)}$. Mesmo com a expansão de literatura sobre o PS nos últimos anos, constata-se que, ainda, há a necessidade de realização de pesquisas que demonstrem a aplicação de modelos de planejamento e de desenvolvimento de lideranças, comprovando melhores práticas ${ }^{(3)}$.

Com base nessa lacuna importante, foi proposto o presente estudo, fundamentado na urgente necessidade de criar estratégias que garantam uma força de trabalho em enfermagem qualificada, para ocupar os papéis de liderança. A falta de evidências sobre as melhores práticas para o desenvolvimento de líderes nas organizações motivou a elaboração deste estudo, cujo objetivo foi descrever a construção de um PS de lideranças de enfermagem para uma instituição hospitalar, com base na reflexão apreciativa de enfermeiros.

\section{MÉTODO}

\section{Desenho do estudo}

Estudo descritivo, exploratório, de abordagem qualitativa, fundamentado na Investigação Apreciativa (IA $)^{(14)}$, cuja abordagem se propõe a destacar "o que há de melhor, o que dá vida a um sistema em seu estado mais eficaz e capaz, em termos econômicos, sociais, ecológicos e humanos"(15). A IA pode ser definida como "a busca cooperativa, coevolucionária pelo melhor das pessoas e suas organizações e pelo mundo que as rodeia" ${ }^{\prime 16)}$, pois valoriza o que há de positivo em determinada situação. Caracteriza-se pela investigação da multiplicidade e da potencialidade das relações humanas, baseada em pontos fortes para o desenvolvimento e gestão de mudanças da organizaçã̃o ${ }^{(17)}$. A utilização da IA vem ganhando destaque no contexto da saúde ${ }^{(18-20)}$, por facilitar o engajamento da força de trabalho, a promoção da aprendizagem organizacional e as mudanças organizacionais positivas ${ }^{(20-21)}$.

Para o desenvolvimento do estudo, foram seguidas as orientações do Consolidated criteria for reporting qualitative research (COREQ): a 32-item checklist for interviews and focus groups $^{(22)}$ na composição de cada uma das etapas, com vistas a alcançar o máximo rigor possível no decorrer da investigação.

\section{Critérios de SeleÇão}

A pesquisa foi realizada em um hospital público federal de ensino de uma capital do Centro-Oeste brasileiro, no ano de 2017. O hospital é caracterizado como sendo geral, que presta atendimento ambulatorial e hospitalar, em nível de atenção básica, de média e de alta complexidade, aos usuários do Sistema Único de Saúde (SUS).

Os participantes incluídos na pesquisa foram enfermeiros com, no mínimo, um ano de trabalho na instituição, que atuavam em diversos setores e tinham diferentes níveis hierárquicos. O recrutamento dos participantes ocorreu após a mobilização dos profissionais, divulgando-se a temática, e contou com o apoio institucional e da Divisão de Enfermagem, que incluiu a atividade na escala de trabalho daqueles envolvidos. Para esse processo, foi utilizada a técnica de "bola de neve" (snow-ball) ${ }^{(23)}$, em que, para cada enfermeiro convidado era-lhe solicitado indicar outros dois profissionais que pudessem participar. Foram excluídos aqueles enfermeiros que, apesar de terem aderido ao convite, não conseguiram cumprir o cronograma da construção do planejamento ou afastaram-se das suas atividades no decorrer da pesquisa por qualquer motivo (férias, licença-médica, rescisão de contrato com a instituição). No primeiro contato com os participantes, foram apresentados o objetivo e as etapas do estudo e solicitada a assinatura do Termo de Consentimento Livre e Esclarecido. 
Participaram da primeira etapa desse estudo 36 enfermeiros, que passaram pela entrevista individual. Estes foram convidados a participar da segunda etapa da pesquisa por meio de um convite impresso, entregue pessoalmente pela pesquisadora. Nessa oportunidade, solicitou-se a confirmação dos interessados para participar de um encontro grupal para fins de construção do planejamento, assim, participaram dessa segunda etapa 22 enfermeiros.

\section{Coleta de DAdos}

A coleta de dados ocorreu em duas etapas, sendo a primeira por meio de entrevistas individuais, a fim de verificar a experiência relacionada à liderança e suas perspectivas sobre a sucessão de lideranças de enfermagem. Para a realização da entrevista, foi utilizado um roteiro que continha 25 perguntas relacionadas com caracterização pessoal e profissional dos participantes, experiência dos enfermeiros quanto à liderança, e questões sobre a sucessão de liderança elaboradas com base nos elementos básicos do PS.

As entrevistas foram agendadas previamente, de acordo com a disponibilidade dos participantes, e realizadas nas dependências do hospital, em local reservado, nos respectivos setores de atuação dos entrevistados. Todas foram gravadas após o consentimento dos entrevistados.

A coleta de dados da segunda etapa foi conduzida com base nos pressupostos da $\mathrm{IA}^{(15)}$ que, operacionalmente, concretiza-se por meio do Ciclo 4D, em que são trabalhados quatro aspectos: descoberta, sonhos, planejamento e destino (em inglês: Discovery, Dream, Design e Destiny). Para cada um desses aspectos, são realizadas perguntas apreciativas que instigam mudanças nas pessoas ao estimulá-las a pensar, discutir e falar sobre o que está sendo proposto e, consequentemente, vislumbrar o futuro a ser construído ${ }^{(16)}$.

No presente estudo, esse processo foi mediado por uma intervenção grupal, com duração de cinco horas, conduzida por equipe com formação específica sobre a IA e o Ciclo 4D. Além disso, a intervenção foi coordenada por três das pesquisadoras, todas com formação em Dinâmica de Grupo, e uma delas com formação em Coaching.

O preparo do grupo para a intervenção e sua mobilização para a construção do planejamento foi feito por meio de uma exploração das impressões e perspectivas dos profissionais sobre as iniciativas institucionais no preparo de novas lideranças, por meio de entrevistas individuais na primeira etapa do estudo. Foram mapeadas as potencialidades da liderança em enfermagem na instituição, as necessidades de capacitação para o enfrentamento da sucessão de lideranças e a disponibilidade dos enfermeiros para a construção do PS. Os resultados desse mapeamento apontaram que o grupo tinha disponibilidade e potencial para o desenvolvimento desse $\mathrm{PS}^{(21)}$.

A intervenção grupal ocorreu em espaço próximo ao hospital do estudo, em uma sala reservada e com condições para concentração do grupo e melhor desempenho coletivo. Para o aquecimento desses enfermeiros, foram apresentados os resultados do mapeamento da entrevista individual feita anteriormente com eles, momento em que foram apreendendo a sua própria visão acerca das impressões e das perspectivas para a sucessão de lideranças, em nível institucional.

Em seguida, o grupo foi dividido em cinco subgrupos, para o cumprimento das quatro etapas da estrutura metodológica do Ciclo 4D, esquematizadas no Quadro 1.

Quadro 1 - Representação esquemática das etapas da coleta de dados feita com enfermeiros de um hospital público federal de ensino utilizando o Ciclo do 4D da Investigação Apreciativa - Goiânia, GO, Brasil, 2017.

\begin{tabular}{|c|c|c|}
\hline Fases & Estratégia & Pergunta \\
\hline $\begin{array}{l}1^{\circ} \mathrm{D} \\
\text { Descoberta }\end{array}$ & $\begin{array}{l}\text { Cada subgrupo recebeu três filipetas para apontar três pontos } \\
\text { positivos que acreditava existir na instituição, relacionados à } \\
\text { sucessão de liderança. }\end{array}$ & $\begin{array}{c}\text { "Com base em sua vivência, quais pontos positivos } \\
\text { você identifica para a sucessão de liderança na } \\
\text { instituição?" }\end{array}$ \\
\hline $\begin{array}{l}2^{\circ} \mathrm{D} \\
\text { Sonho }\end{array}$ & $\begin{array}{l}\text { Considerando os pontos positivos identificados pelos grupos, foi } \\
\text { solicitado, na sequência, que cada subgrupo descrevesse cinco } \\
\text { sonhos relacionados à sucessão de liderança para a instituição. } \\
\text { Todas as descrições foram coladas no quadro-negro. }\end{array}$ & $\begin{array}{l}\text { "Quais são os seus sonhos para um futuro ideal com } \\
\text { relação à sucessão de liderança nessa instituição?" }\end{array}$ \\
\hline $\begin{array}{l}3^{\circ} \mathrm{D} \\
\text { Planejamento }\end{array}$ & $\begin{array}{l}\text { Tendo os sonhos do grupo todo descritos, foi solicitada, então, a } \\
\text { seleção daqueles considerados passíveis de realização. Com os } \\
\text { sonhos definidos, foi elaborado um quadro, com visualização de } \\
\text { todos, para registro das metas e prazos. }\end{array}$ & $\begin{array}{l}\text { "Quais sonhos são possíveis de serem alcançados? } \\
\text { Quais metas precisam traçar para atingir esses } \\
\text { sonhos? Qual o tempo para alcançar essas metas?" }\end{array}$ \\
\hline $\begin{array}{l}4^{\circ} \mathrm{D} \\
\text { Destino }\end{array}$ & $\begin{array}{l}\text { Para a concretização do planejamento, na etapa Destino, foi ainda } \\
\text { elaborado o que precisava ser feito para alcançar cada meta, bem } \\
\text { como as ações necessárias e seus responsáveis por executá-las. }\end{array}$ & $\begin{array}{l}\text { "O que precisa ser feito para que os sonhos se } \\
\text { realizem?" "Quais ações precisam ser feitas } \\
\text { para que os sonhos se realizem?" "De quem é a } \\
\text { responsabilidade por realizar os sonhos?" }\end{array}$ \\
\hline
\end{tabular}

Como descrito no Quadro 1, na construção do planejamento, as perguntas relativas ao primeiro e segundo "D" foram direcionadas aos subgrupos com tempo para respostas, de modo que os participantes discutiram, entraram em consenso, elaboraram e compartilharam, finalmente, suas respostas com o grupo. Para o registro do consenso das respostas, foram entregues para cada subgrupo filipetas de papel que foram anexadas ao quadro negro da sala, no momento da apresentação oral dos subgrupos. Essas filipetas ficaram coladas no quadro, de forma que o PS ficou ilustrado, em sua completude, à disposição do grupo.

As perguntas relacionadas ao terceiro e quarto "D" foram direcionadas a todos os envolvidos, que construíram suas respostas coletivamente, ilustrando o esboço do planejamento em um quadro visualizado por todos. 
O registro dos dados nesta etapa ocorreu por meio de anotações realizadas em um diário de campo, durante o processo grupal, pelas pesquisadoras, do material produzido coletivamente durante o encontro grupal e por meio de fotografias.

\section{ANÁlISE E TRATAMENTO DOS DADOS}

As entrevistas individuais da primeira etapa foram transcritas, lidas exaustivamente e, posteriormente, analisadas para codificação e categorização do material. Para tanto, contou-se com o auxílio do WebQDA 2016, software utilizado para analisar textos, vídeos, áudios e imagens, pertinente à investigação qualitativa baseada na codificação das informações.

Para apresentação dos dados da primeira etapa, foram criados códigos referentes a cada participante do estudo. Para elaboração do código, utilizou-se a primeira letra do cargo ocupado pelo enfermeiro na instituição, seguida de um número correspondente à ordem de transcrição das entrevistas. Para a padronização do código, como dito anteriormente, optou-se por agrupar enfermeiros gerentes, chefes de divisão e chefes de setores, denominados "diretores", por estarem diretamente ligados à diretoria da instituição, identificando-os, portanto, pelo código "D”(D1 a D5). Aos chefes de unidades atribuiu-se o código " $\mathrm{C}$ " $(\mathrm{C} 1$ a $\mathrm{C} 8)$, aos líderes, "L" (L1 a L7) e aos supervisores, "S" (S1 a S16).

A escolha dos dados das entrevistas a serem socializados na segunda etapa da pesquisa baseou-se nos princípios da IA, de modo a possibilitar que a proposta de PS de lideranças de enfermagem para o hospital fosse uma coconstrução e fizesse sentido para as pessoas envolvidas. No caso, selecionou-se, das entrevistas, o conteúdo relacionado à visão positiva do grupo acerca da liderança e do processo sucessório.

O registro dos dados produzidos na intervenção foi feito pela anotação e pelas fotografias do planejamento projetado no quadro, além de notas de campos sobre o processo grupal e a construção do material produzido, coletivamente, na intervenção. A análise dos dados foi feita de forma descritiva, a partir do processo vivido pelo grupo na intervenção, articulada criticamente com a literatura especializada.

\section{Aspectos Éticos}

O projeto que deu origem à pesquisa obteve parecer favorável do Comitê de Ética em Pesquisa do hospital onde foi realizado, no ano de 2016, CAAE $n^{\circ}$ 54854716.9.0000.5078, Parecer $\mathrm{n}^{\circ} 1.500 .459$.

\section{RESULTADOS}

\section{CARACTERIZAÇ̃̃O DOS PARTICIPANTES}

Participaram da intervenção, para construção do PS para o hospital, 22 enfermeiros, a maioria do sexo feminino (19/86,4\%), com idade entre 38 e 53 anos (10/45,4\%), de todas as ocupações hierárquicas existentes na instituição (gerente, chefe de divisão, chefe de setor, chefe de unidade, líder de clínica e supervisor de clínica). Todos tinham pós-graduação, a maioria, mestrado (10/45,4\%) e especialização (9/41,0\%), e tempo de formação prevalente entre 26 e 35 anos (8/36,4\%). A seguir, estão apresentados os dados relacionados ao ciclo $4 \mathrm{D}$.

A Fase da Descoberta - $1^{\circ} \mathrm{D}$ apontou os pontos positivos identificados pelos enfermeiros para a sucessão de lideranças na instituição, que são: compromisso; troca de experiências; flexibilidade; estratégia da gestão atual; profissionais empenhados em fazer o melhor; planejamento e motivação para a sucessão de liderança; qualificação dos profissionais e gestores; consciência da necessidade da sucessão de liderança; desenvolvimento de competências; continuidade das ações e conquistas; e envolvimento dos gestores com a sucessão de liderança.

A Fase dos Sonhos - $2^{\circ} \mathrm{D}$ ocupou-se em mapear todos os sonhos considerados pelos enfermeiros, para nortear e alimentar a construção do PS. Os participantes entenderam que eles poderiam ser divididos em dois grandes grupos. $\mathrm{O}$ primeiro, relacionado à política interna e gestão compartilhada, que apresentou quatro sonhos: 1 . Estabelecimento de política interna de desenvolvimento de liderança; 2 . Implantação de uma gestão compartilhada; 3. Maior aproximação da alta gestão com a "ponta" e realização de planejamento participativo; e 4. Compartilhamento de decisões e maior autonomia da equipe; e estão apresentados na sequência. $\mathrm{E}$ o segundo grupo, relacionado ao desenvolvimento de liderança, que elencou cinco sonhos: 1. Criação de programa de formação em liderança - "coaching"; 2. Desenvolvimento, nas lideranças, de atitudes éticas e respeito hierárquico; 3. Motivação dos enfermeiros para a liderança; 4. Efetivação da prática pela análise de indicadores; e 5. Implantação e efetivação do plano para formação de novos líderes.

$\mathrm{Na}$ Fase do Planejamento - $3^{\circ} \mathrm{D}$, o grupo todo vislumbrou o conjunto dos sonhos descritos, definiu metas e aprazamentos para sua realização. Para a primeira categoria de sonhos, relacionados à política interna e à gestão compartilhada, foram traçadas três metas com os respectivos aprazamentos, conforme Quadro 2.

Quadro 2 - Sonhos definidos por enfermeiros de um hospital público federal de ensino relacionados à política interna e gestão compartilhada, com suas respectivas metas e aprazamentos - Goiânia, GO, Brasil, 2017.

\begin{tabular}{|l|c|c|}
\hline \multicolumn{2}{|c|}{ Política interna/Gestão compartilhada } \\
\hline \multicolumn{1}{|c|}{ Sonhos } & Metas & Aprazamento \\
\hline Estabelecimento de política interna de desenvolvimento de liderança & $\begin{array}{c}\text { Estabelecer diretrizes para a } \\
\text { sucessão de lideranças. }\end{array}$ & Um ano \\
\cline { 1 - 2 } Implantação de uma gestão compartilhada & Consolidar uma gestão compartilhada. & Dois anos \\
\cline { 1 - 2 } $\begin{array}{l}\text { Maior aproximação da alta gestão com a "ponta" e realização de } \\
\text { planejamento participativo }\end{array}$ & $\begin{array}{c}\text { Mapear cargos, prever } \\
\text { aposentadorias e identificar } \\
\text { competências. }\end{array}$ & Seis meses \\
\cline { 1 - 2 } Compartilhamento de decisões e maior autonomia da equipe & & \\
\hline
\end{tabular}


Para os sonhos relacionados com o desenvolvimento de liderança, foram definidas duas metas no Quadro 3.
$\mathrm{Na}$ Fase do Destino-4o D, o grupo concluiu o trabalho de desenvolvimento do PS, apresentando como resultado o PS de Enfermagem da instituição, disposto no Quadro 4.

Quadro 3 - Sonhos definidos por enfermeiros de um hospital público federal de ensino relacionados ao desenvolvimento de liderança, com suas respectivas metas e aprazamentos - Goiânia, GO, Brasil, 2017.

\begin{tabular}{|c|c|c|}
\hline \multicolumn{3}{|c|}{ Desenvolvimento da liderança } \\
\hline Sonhos & Metas & Aprazamento \\
\hline Criação de um programa de formação de liderança & \multirow{4}{*}{$\begin{array}{l}\text { Desenvolver um programa de } \\
\text { formação de lideranças. }\end{array}$} & \multirow{4}{*}{ Um ano e meic } \\
\hline Desenvolvimento, nas lideranças, de atitudes éticas e respeito hierárquico & & \\
\hline Motivação dos enfermeiros para a liderança & & \\
\hline Efetivação da prática pela análise de indicadores & & \\
\hline Implantação e efetivação do plano para formação de novos líderes & $\begin{array}{c}\text { Implantar um programa de } \\
\text { desenvolvimento de lideranças. }\end{array}$ & Dois anos \\
\hline
\end{tabular}

Quadro 4 - Planejamento de sucessão construído por enfermeiros de um hospital público federal de ensino - Goiânia, GO, Brasil, 2017.

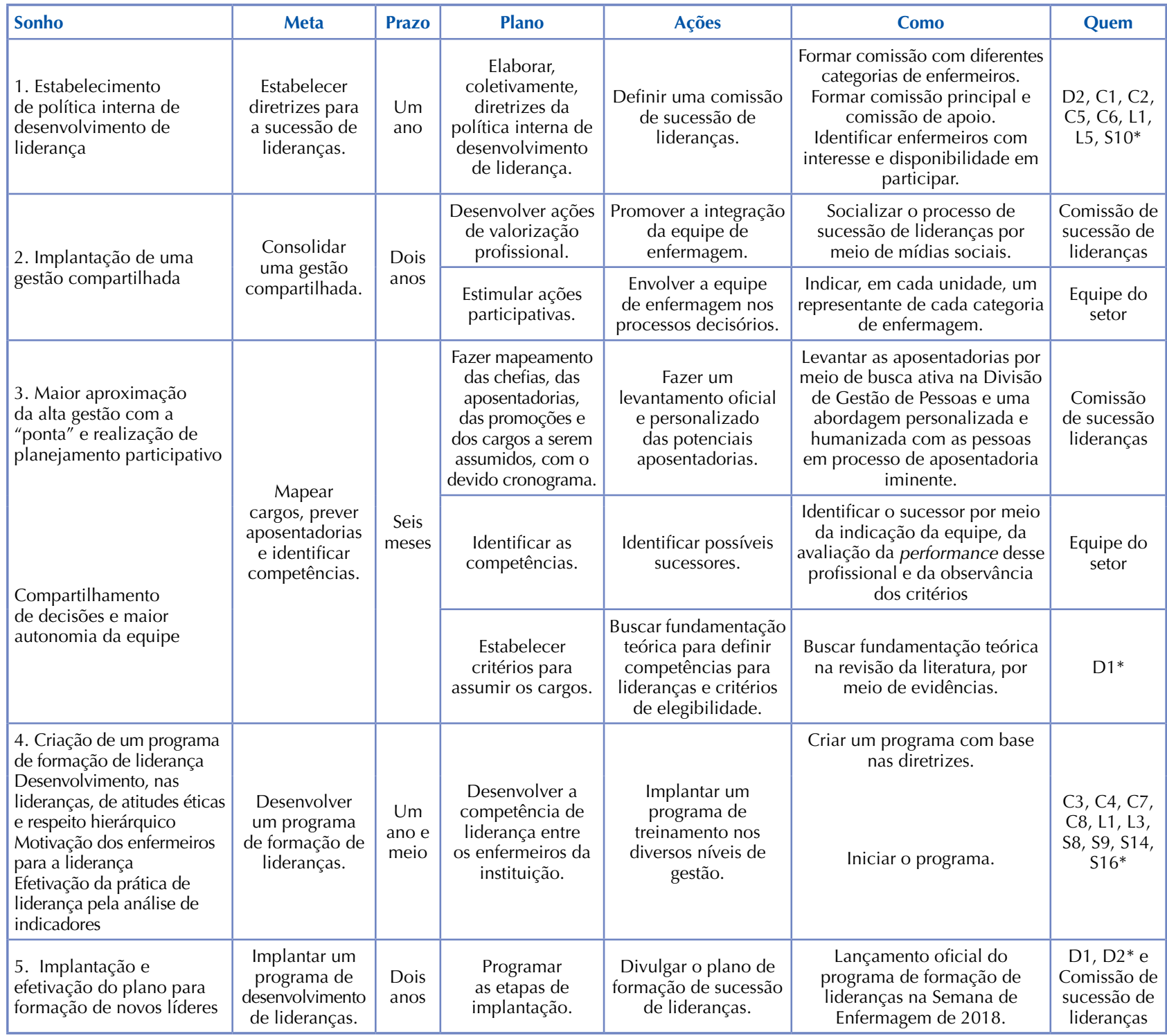

* Os códigos foram elaborados para referir-se a cada participante do estudo, utilizando a primeira letra do cargo ocupado pelo enfermeiro na instituição, seguida de um número correspondente à ordem de transcrição das entrevistas. O Código “D” (D1 a D5) refere-se a enfermeiros gerentes, chefes de divisão e chefes de setores, denominados "diretores"; "C" (C1 a C8) refere-se aos chefes de unidades; "L" (L1 a L8), aos líderes, e "S" (S1 a S16), aos supervisores. 


\section{DISCUSSÃO}

A participação de representantes de todas as categorias de enfermeiros permitiu a construção coletiva de um Planejamento de Sucessão de Lideranças em Enfermagem concebido por meio de discussão ampla, amadurecida e fiel às necessidades institucionais. Garantir a presença de enfermeiros de todos os segmentos hierárquicos é fundamental e recomendado na IA, pois reflete a pluralidade de ideias e opiniões do contexto estudado ${ }^{(15)}$. Além disso, para a construção do PS, a literatura recomenda a representação da opinião de todos os enfermeiros, e não apenas a dos gestores ${ }^{(6)}$.

Analisando os participantes na perspectiva dos estudos intergeracionais, observa-se que, embora a maioria dos participantes pertencesse à geração $X$, nesta etapa também houve o cuidado de assegurar a diversidade geracional e, portanto, havia representantes das gerações $Y$ e baby boomers. $\mathrm{Na}$ elaboração do planejamento, essa diversidade viabiliza que pontos fortes, valores e expectativas de cada geração tenham contribuição ${ }^{(24)}$. Além disso, considerando o tempo de formação, pode-se afirmar que o PS foi construído por pessoas experientes, aspecto que favorece uma visão fundamentada na prática e no desenvolvimento de competências para a liderança $a^{(4,13,25-26)}$.

Os pressupostos e fundamentos da IA facilitaram a construção do PS. A aplicação do Ciclo $4 \mathrm{D}$ permitiu uma abordagem positiva baseada nos pontos fortes da instituição ${ }^{(16)}$, a organização do pensamento, do tempo e do esforço dos participantes. No $1^{\circ} \mathrm{D}$, foi possível observar que os aspectos positivos identificados pelos enfermeiros, como compromisso, flexibilidade, qualificação e empenho em fazer o melhor, apontam para facilitadores da liderança. Estes são definidos, na literatura, como requisitos pessoais e profissionais de líderes de enfermagem autênticos e bem-sucedidos ${ }^{(10,27)}$.

Os resultados do trabalho no $2^{\circ} \mathrm{D}$ apontaram que os sonhos definidos pelo grupo remetem, indiretamente, aos elementos que compõem o modelo de PS proposto por um estudo pioneiro sobre esse assunto ${ }^{(28)}$, a saber: planejamento estratégico, identificação de habilidades desejadas (competência), identificação de posição-chave, seleção de candidatos, orientação e coaching, processos de desenvolvimento, alocação de recursos e avaliação. Apesar de os sonhos apresentados focarem o desenvolvimento de liderança, planejamento estratégico e coaching, fazem referência às especificidades e às necessidades percebidas pelos enfermeiros como importantes para a instituição.

A partir dos sonhos elencados, nas fases $3^{\circ} \mathrm{D}$ e $4^{\circ} \mathrm{D}$, os participantes focaram aquilo que têm capacidade de alcançar enquanto grupo, para a situação específica vivida na organização. Neste caso, o PS, resultado final do $4^{\circ} \mathrm{D}$, foi elaborado coletivamente pelos enfermeiros e abordou três eixos principais: política interna da instituição, com foco na gestão compartilhada e participativa; criação de um programa de desenvolvimento de liderança; e implantação do PS na instituição.

A gestão compartilhada e participativa como política interna de uma instituição possibilita ambiente de trabalho inovador e transformador, pois incentiva a atualização e a transmissão dos conhecimentos ${ }^{(29)}$. As representações de diferentes categorias da enfermagem nos processos decisórios, a elaboração de uma comissão de sucessão e a divulgação por meio de mídias sociais sobre as etapas do processo sucessório podem atrair enfermeiros a posições de lideranças, por permitir compartilhamento e trocas de experiências entre profissionais em diferentes etapas da carreira ${ }^{(8,26,30)}$.

Os dados gerados no presente estudo indicaram que a gestão compartilhada é percebida por enfermeiros, de diferentes níveis hierárquicos, como um instrumento que facilita a compreensão dos papéis de liderança, dos processos de tomada de decisão e cria uma cultura de compartilhamento que permite o envolvimento de uma diversidade de pessoas nos processos administrativos do hospital, possibilitando que jovens enfermeiros também exercitem papéis de liderança.

O segundo ponto abordado no PS elaborado nesta pesquisa foi a criação de um programa de desenvolvimento de lideranças. Preparar futuros líderes de enfermagem contribui, sobremaneira, para a sucessão de lideranças e o futuro da enfermagem, pois essas pessoas poderão dar continuidade à gestão ${ }^{(13)}$, o que é fundamental para o sucesso das organizações de saúde ${ }^{(4)}$.

Apesar da importância do desenvolvimento de lideranças para o PS, não há ainda a aplicação de modelos de desenvolvimento de lideranças com comprovação de melhores práticas $^{(3)}$. Porém, estudos recentes indicam que: a organização de estratégias que permitam a troca de experiências e a imersão nos papéis de liderança ${ }^{(4,8)}$; a definição dos papéis, as descrições e as expectativas dos cargos $^{(5,10)}$; o mapeamento de carreiras $^{(5)}$; e os programas robustos de mentoria ${ }^{(4,8,10)}$ podem auxiliar em programas de desenvolvimento de lideranças.

Embora as evidências científicas apontem caminhos ainda incipientes em relação às melhores práticas para $o$ desenvolvimento de lideranças, entende-se que estes devem ser orientados pela necessidade da instituição, de maneira personalizada e, por isso, devem ser construídos coletivamente. Nesse sentido, é fundamental o conhecimento dos diversos tipos de PS e sua adequação e pertinência a cada tipo de instituição onde serão aplicados, bem como a cultura, os valores e as prioridades organizacionais ${ }^{(6,9)}$.

Vale destacar um dado relacionado no PS construído, sonho três, sobre a organização do planejamento participativo, a respeito da contribuição dos líderes experientes e o cuidado que se deve ter ao se tratar da proximidade de suas aposentadorias. Esses profissionais, representados pela geração baby boomers, nascidos entre 1946 e 1964, têm como características o fato de serem ativos, muito trabalhadores e envolvidos e menos influenciáveis pelos aspectos negativos do trabalho, com menor intenção de deixar o emprego ${ }^{(24)}$.

Por essa razão, em respeito ao trabalho desse grupo, ficou explícito, no planejamento, que a comissão responsável lidasse com aqueles que, em breve, deixariam seus cargos, por meio de uma abordagem personalizada e humanizada no processo de mapeamento de tempo de aposentadorias. Isso sugere que o grupo se preocupou em deixar registrada a importância de tratar, de forma respeitosa, a situação daqueles que dedicaram anos de trabalho à instituição, ao mesmo tempo em que podem prever quantos não mais estarão trabalhando no futuro a curto e médio prazos. 
Dessa forma, o mapeamento de carreiras ultrapassa o simples registro das competências e das necessidades que cada cargo exige, o que é essencial para o desenvolvimento de lideranças orientado para as necessidades do hospital. Porém, traz um adicional relacionado ao cuidar dos mais experientes, o que torna esse dado uma evidência rara nos estudos publicados.

Nesse mesmo sonho, destaca-se, ainda, o cuidado do grupo em buscar estratégias voltadas não só para além de indicações das chefias para a ocupação de cargos de liderança, mas também considerar a contribuição das equipes na indicação de possíveis sucessores. Além disso, é evidente a preocupação em atender às necessidades das gerações $Y$ e $Z$ por capacitação e desenvolvimento de programas estruturados de desenvolvimento de liderança ${ }^{(24)}$. Nesse sentido, também é possível observar que o sonho relacionado a esse aspecto é detalhado com destaque à necessidade de formação com base ética e fundamentada nas melhores evidências da literatura, o que sugere que o grupo apresenta maturidade em escolher caminhos seguros na condução do processo sucessório.

A escolha da IA na condução da construção do PS em Enfermagem do hospital em questão deu luz aos pontos positivos do contexto institucional e do grupo envolvido. Esse aspecto favorece que a execução do PS e sua forma de aplicação sejam concebidas com base nos valores e prioridades da instituição. Consequentemente, a proposta possivelmente refletirá a missão e o plano estratégico da organização ${ }^{(9)}$.

As perguntas apresentadas, estrategicamente, para que se buscasse uma perspectiva positiva do trabalho institucional (princípio positivo), mobilizaram mudanças (princípio da simultaneidade) para o fortalecimento das capacidades e desenvolvimento das potencialidades (princípio antecipatório), o que promoveu a construção de um plano possível para as condições institucionais, criado a partir da colaboração daqueles que o executarão no futuro (princípio construcionista) ${ }^{(16)}$.

Nesse sentido, o PS construído apresentou ao grupo que o concebeu uma importante ferramenta para potencializar o desenvolvimento de liderança no hospital, refletindo os anseios dos profissionais por um processo organizado de continuidade da liderança, focado na qualificação e em um ambiente colaborativo e comprometido com a melhoria da assistência de enfermagem.

Sob o olhar dos enfermeiros, a instituição apresentou pontos favoráveis para a implantação do PS de lideranças, uma vez que há consciência e envolvimento dos gestores em relação a esse propósito, além de planejamento e motivação dos próprios enfermeiros para a sucessão. Além disso, a escolha do foco na gestão participativa possibilitou ao PS em enfermagem da instituição atuar como um grande agente de mudança na política interna, por estimular a implementação de processos semelhantes em outras áreas da instituição.

Os princípios da IA (positivo, construtivista, de simultaneidade, poético e antecipatório) utilizados no decorrer das etapas do ciclo 4D, possivelmente, favoreceram a elaboração do referido PS de Enfermagem. Há de se ressaltar que a postura dos enfermeiros participantes da pesquisa foi de colaboração e cuidado uns com os outros, em uma perspectiva participativa e de pertencimento, durante a construção do planejamento.

Finalmente, destacamos que os resultados apontados no PS construído constituem-se em uma síntese de importante avanço na construção do conhecimento, por possibilitar um olhar sistêmico de um planejamento sucessório desenvolvido à luz da IA. A união desses dois referenciais possibilitou uma perspectiva inovadora à abordagem do tema no contexto de organizações hospitalares, podendo estimular outros pesquisadores e gestores no enfrentamento desse grande desafio atual. Como limitações do estudo apontamos a especificidade da cultura organizacional que pode restringir a generalização dos resultados. Ainda, tendo em vista o cronograma de realização da pesquisa, não foi possível o cumprimento dos passos de implementação do planejamento e seu acompanhamento, o que poderá ser feito por meio de outras investigações futuras.

\section{CONCLUSÃO}

Este estudo, realizado na perspectiva teórico-metodológica da IA e considerando as características da organização na qual se inserem os diversos atores, enfermeiros assistenciais e gestores, culminou na proposta de um PS. O produto final integrou com êxito os fundamentos teóricos da metodologia utilizada, e os princípios adotados permitiram que o planejamento construído fosse capaz de potencializar ações de desenvolvimento de liderança no hospital. O ambiente colaborativo entre gerações foi valorizado pelos participantes, e um planejamento com foco na gestão participativa foi construído. Esse aspecto pode alterar a política interna da instituição em diversas áreas e apontar caminhos para a implementação do PS em diferentes contextos.

\section{RESUMO}

Objetivo: Descrever a construção de um planejamento de sucessão de lideranças de enfermagem para uma instituição hospitalar, com base na reflexão apreciativa de enfermeiros. Método: Estudo descritivo, exploratório, de abordagem qualitativa realizada com enfermeiros de um hospital público federal de ensino. A coleta de dados foi realizada em duas etapas, sendo a primeira, entrevista individual e a segunda, por mediação grupal conduzida com base no ciclo 4D e nos pressupostos da Investigação Apreciativa. Foi realizada análise descritiva dos achados. Resultados: Os dados apontaram para uma equipe de diferentes gerações e altamente qualificada, preocupada com um planejamento de sucessão baseado em evidências científicas, que tenha envolvimento das equipes, e realizado, de maneira cuidadosa, para os enfermeiros em diferentes etapas da carreira profissional. Conclusão: Os princípios da Investigação Apreciativa permitiram a construção de um planejamento de sucessão para potencializar ações de desenvolvimento de liderança no hospital. Nesse processo, o ambiente colaborativo entre gerações foi valorizado e um planejamento com foco na gestão participativa foi construído. Esse aspecto pode alterar, profundamente, a política interna da instituição em diversas áreas e apontar caminhos para a implementação do planejamento de sucessão em diferentes contextos.

\section{DESCRITORES}

Pesquisa em Administração de Enfermagem; Liderança; Educação Continuada em Enfermagem; Mão de Obra em Saúde; Enfermeiros Administradores; Pesquisa Qualitativa. 


\section{RESUMEN}

Objetivo: Describir la construcción de una planificación de sucesión de liderazgos de enfermería para una institución hospitalaria, con base en la reflexión apreciativa de enfermeros. Método: Estudio descriptivo, exploratorio, de abordaje cualitativo, realizado con enfermeros de un hospital público de enseñanza. Datos recolectados en dos etapas: la primera consistió en una entrevista individual; la segunda fue por mediación grupal dirigida en base a los ciclos 4D y los supuestos de la Investigación Apreciativa. Se realizó análisis descriptivo de los hallazgos. Resultados: Los datos describieron un equipo con miembros de diferentes generaciones, altamente calificado, preocupado con una planificación sucesoria basada en evidencias científicas, con involucramiento de los equipos y realizado de manera cuidadosa para los enfermeros en distintas etapas de su carrera profesional. Conclusión: Los principios de la Investigación Apreciativa permitieron construir una planificación sucesoria que impulse acciones de desarrollo y liderazgo en el hospital. En este proceso fue valorado el ambiente colaborativo entre generaciones, y se elaboró una planificación con foco en la gestión. Dicho aspecto puede cambiar significativamente la política interna de la institución en diversas áreas y señalar caminos para la implementación de la planificación de sucesiones en diferentes contextos.

\section{DESCRIPTORES}

Investigación en Administración de Enfermería; Liderazgo; Educación Continua en Enfermería; Fuerza Laboral en Salud; Enfermeras Administradoras; Investigación Cualitativa.

\section{REFERÊNCIAS}

1. Ross C, Olson JK, Kushner KE, Murad SS, Leung WSW, Daniels S, et al. Student preparation for nursing leadership: lessons from an undergraduate programs review. Int J Nurs Educ Scholarsh. 2018;15(1). https://doi.org/10.1515/ijnes-2017-0039

2. Warshawsky N, Cramer E. Describing nurse manager role preparation and competency: findings from a national study. JONA 2019;49(5):249-55. https://doi.org/10.1097/NNA.0000000000000746

3. LaCross E, Hall N, Boerger JA. Nurse manager succession planning: evaluating a pilot program's effect on self-perception of readiness. JONA 2019;49(6):331-5. https://doi.org/10.1097/NNA.0000000000000761

4. Ramseur P, Fuchs MA, Edwards P, Humphreys J. The implementation of a structured nursing leadership development program for succession planning in a health system [Internet]. J Nurs Adm. 2018;48(1):25-30. https://doi.org/10.1097/NNA.0000000000000566

5. Webb T, Diamond-Wells T, Jeffs D. Career mapping for professional development and succession planning. J Nurses Prof Dev. 2017;33(1):25-32. https://doi.org/10.1097/NND.0000000000000317

6. Scholes J, Trapani J. Succession planning: a case for revisiting the process in critical care. Nurs Crit Care. 2017;22(4):193-4. https://doi.org/10.1111/nicc. 12308

7. Titzer JL, Shirey MR, Hauck S. A nurse manager succession planning model with associated empirical outcomes, J Nurs Adm 2017;47(10):4958. https://doi.org/10.1097/01.NNA.0000525958.92512.ce

8. Pedersen A, Sorensen J, Babcock T, Bradley M, Donaldson N, Donnelly JE, et al. A nursing leadership immersion program: succession planning using social capital. J Nurs Adm. 2018 Mar;48(3):168-74. https://doi.org/10.1097/NNA.0000000000000592

9. Phillips T, Evans JL, Tooley S, Shirey MR. Nurse manager succession planning: a cost-benefit analysis. J Nurs Manag. 2018;26(2):238-43. https://doi.org/10.1111/jonm.12512

10. Fray B, Sherman RO. Best practices for nurse leaders: succession planning. Prof Case Manag. 2017;22(2):88-94. https://doi.org/10.1097/ NCM.0000000000000214

11. Sessler Branden P, Sharts-Hopko NC. Growing clinical and academic nursing leaders: building the pipeline. Nurs Adm Q. 2017;41(3):258-65. https://doi.org/10.1097/NAQ.0000000000000239

12. Andrigue KC, Trindade LL, Amestoy SC. Academic formation and permanent education: influences on leadership styles of nurses. J Res Fundam Care. 2017;9(4):971-7. https://doi.org/10.9789/2175-5361.2017.v9i4.971-977

13. Amestoy SC, Trindade LL, Silva GT, Santos BP, Reis VR, Ferreira VB. Leadership in nursing: from teaching to practice in a hospital environment. Esc Anna Nery. 2017;21(4):e20160276. https://doi.org/10.1590/2177-9465-ean-2016-0276

14. Cooperrider DL, Srivastva S. Appreciative inquiry in organizational life. In: Pasmore WA, Woodman RW, editors. Research in organizational change and development. Greenwich: JAI Press; 1987. p. 129-69.

15. Cooperrider DL, Whitney D. Appreciative inquiry: a positive revolution in change. Oakland: Berrett-Koehler; 2005.

16. Cooperrider DL, Whitney D. Investigacao apreciativa: uma abordagem positiva para a gestao de mudancas. Rio de Janeiro: Qualitymark; 2006.

17. Magnussen IL, Alteren J, Bondas T. Appreciative inquiry in a Norwegian nursing home: a unifying and maturing process to forward new knowledge and new practice. Int J Qual Stud Health Well-being. 2019;14(1):1559437. https://doi.org/10.1080/17482631.2018.1559437

18. Burns E, Triandafilidis Z, Schmied V. Designing a model of breastfeeding support in Australia: an appreciative inquiry approach. Health Soc Care Community. 2020;28(5):1723-33. https://doi.org/10.1111/hsc.12997

19. Nease DE, et al. Making the random the usual: appreciative inquiry/boot camp translation-developing community-oriented evidence that J Prim Care Community Health. 2020;11: 2150132720904176. https://doi.org/10.1177/2150132720904176

20. Bleich MR, Hessler C. Appreciative Inquiry and Implementation Science in Leadership Development. J Contin Educ Nurs. 2016 May;47(5):207-9. https://doi.org/10.3928/00220124-20160419-04

21. Nogueira AL, Munari DB, Ribeiro LC, Bezerra AL, Chaves LD. Nurses expectations about the succession of leaders in the hospital context. Rev. Latino-Am Enfermagem 2019;27:e3178. https://doi.org/10.1590/1518-8345.2833.3178.

22. Tong A, Sainsbury P, Craig J. Consolidated criteria for reporting qualitative research (COREQ): a 32-item checklist for interviews and focus groups. Int J Qual Health Care. 2007;19(6):349-57. https://doi.org/10.1093/intqhc/mzm042 
23. Kirchherr J, Charles K. Enhancing the sample diversity of snowball samples: Recommendations from a research project on anti-dam movements in Southeast Asia. PLoS One 2018;13(8). https://doi.org/10.1371/journal.pone.0201710

24. Stevanin S, Palese A, Bressan V, Vehvilainen-Julkunen K, Kvist T. Workplace-related generational characteristics of nurses: a mixed-method systematic review. J Adv Nurs. 2018;74(6):1245-63. https://doi.org/10.1111/jan.13538

25. Martins BG, Silva LMC, Capaccioli BRBS, Neves VR, Balsanelli AP. Development and validation of a leadership training program for nurses. Texto Contexto Enferm. 2019;28:e20180048. https://doi.org/10.1590/1980-265x-tce-2018-0048

26. Munari DB, Nogueira ALG, Sousa ET, Ribeiro LCM, Sherman R. Leadership succession: a necessary reflection for the future of nursing. Rev Eletr Enf. 2019;21:54787-8. https://doi.org/10.5216/ree.v21.54787

27. Delamater L, Hall N. Charge nurse development: what does the literature say? Nurs Manage. 2018;49(7):34-40. https://doi.org/10.1097/01. NUMA.0000538914.53159.fC

28. Carriere BK, Muise M, Cummings G, Newburn-Cook C. Healthcare succession planning: an integrative review. J Nurs Adm. 2009;39(12):548-55. https://doi.org/10.1097/NNA.0b013e3181c18010

29. Stab N, Hacker W. Participatory redesign of work organisation in hospital nursing: a study of the implementation process. J Nurs Manag. 2018;26(4):382-392. https://doi.org/10.1111/jonm.12545

30. Cziraki K, Read E, Spence Laschinger HK, Wong C. Nurses' leadership self-efficacy, motivation, and career aspirations. Leadersh Health Serv (Bradf Engl). 2018;31(1):47-61. https://doi.org/10.1108/LHS-02-2017-0003 